\title{
MAXIMAL SUBALGEBRA OF DOUGLAS ALGEBRA
}

\author{
CARROLL J. GULLORY \\ Department of Mathematics \\ University of Southwestern Louisiana \\ Lafayette, Louisiana 70504 \\ (Received April 1, 1987)
}

\begin{abstract}
When $q$ is an interpolating Blaschke product, we find necessary and sufficient conditions for a subalgebra $B$ of $H^{\infty}[\bar{q}]$ to be a maximal subalgebra in terms of the nonanalytic points of the noninvertible interpolating Blaschke products in $B$. If the set $M(B) \cap Z(q)$ is not open in $Z(q)$, we also find a condition that guarantees the existence of a factor $q_{0}$ of $q$ in $H^{\infty}$ such that $B$ is maximal in $H^{\infty}[\bar{q}]$. We also give conditions that show when two arbitrary Douglas algebras $A$ and $B$, with $A \subseteq B$ have property that $A$ is maximal in $B$.
\end{abstract}

KEY WORDS AND PHRASES. Maximal subalgebra, Douglas algebra, interpolating sequence, sparse sequence, Blaschke product, inner functions, open and closed subset, nonanalytic points, support set, Q-C level sets.

1980 AMS SUBJECT CLASSIFICATION CODE. 46J15, 46J20.

\section{INTRODUCTION.}

Let $\mathrm{D}$ be the open unit disk in the complex plane and $\mathrm{T}$ be its boundary. Let $\mathrm{L}^{\infty}$ be the space of essentially measurable functions on $\mathrm{T}$ with respect to the Lebesgue measure. By $\mathrm{H}^{\infty}$ we mean the family of all bounded analytic functions in D. Via identification with boundary functions, $\mathrm{H}^{\infty}$ can be considered as a uniformly closed subalgebra of $L^{\infty}$. A uniformly closed subalgebra $B$ between $\mathrm{H}^{\infty}$ and $\mathrm{L}^{\infty}$ is called a Douglas algebra. If we let $C$ be the family of continuous functions on $T$, then it is well known that $\mathrm{H}^{\infty}+\mathrm{C}$ is the smallest Douglas algebra containing $\mathrm{H}^{\infty}$ properly. For any Douglas algebra $\mathrm{B}$, we denote by $M(B)$ the space of nonzero multiplicative linear functionals on $B$, that is, the set of all maximal ideals in $B$. An algebra $B_{0}$ is said to be a maximal subalgebra of $B$, if $\mathrm{B}_{1}$ is another algebra with the property that $\mathrm{B}_{0} \subseteq \mathrm{B}_{1} \subseteq \mathrm{B}$, then either $\mathrm{B}_{1}=\mathrm{B}_{0}$ or $\mathrm{B}_{1}=\mathrm{B}_{\text {. }}$

An interpolating sequence $\left\{z_{n}\right\}_{n=1}^{\infty}$ is a sequence in $D$ with the property that for any bounded sequence of complex numbers $\left\{\lambda_{n}\right\}_{n=1}^{\infty}$, there exists $f$ in $H^{\infty}$ such that $f\left(z_{n}\right)=\lambda_{n}$ for all $n$. A well-known condition states that a sequence $\left\{z_{n}\right\}_{n=1}^{\infty}$ is interpolating if and only if

$$
\inf \prod_{n \neq m}^{\infty}\left|\frac{z_{m}-z_{n}}{1-\bar{z}_{n} z_{m}}\right|=\delta>0 .
$$


A Blaschke product

$$
q(z)=\prod_{n=1}^{\infty} \frac{\left|z_{n}\right|}{z_{n}}\left(\frac{z-z_{n}}{1-\bar{z}_{n} z}\right)
$$

is called an interpolating Blaschke product if its zero set $\left\{z_{n}\right\}_{n=1}^{\infty}$ is an interpolating sequence $\left(\left|z_{n}\right| / z_{n} \equiv 1\right.$ is understood whenever $\left.z_{n}=0\right)$. A sequence $\left\{z_{n}\right\}_{n=1}^{\infty}$ is said to be sparse if it is an interpolating sequence and

$$
\lim _{n \rightarrow \infty} \prod_{n \neq m}\left|\frac{z_{m}-z_{n}}{1-z_{n} z_{m}}\right|=1
$$

For a function $q$ in $H^{\infty}+C$, we let $Z(q)=\left\{m \in M\left(H^{\infty}+C\right): q(m)=0\right\}$ be the zero set of $\phi$ in $M\left(H^{\infty}+C\right)$. An inner function is a function in $H^{\infty}$ of modulus 1 almost everywhere on $T$. We denote by $\mathrm{H}^{\infty}[\overline{\mathrm{b}}]$ the Douglas algebra generated by $\mathrm{H}^{\infty}$ and the complex conjugate of the inner function $b$.

We put $X=M\left(L^{\infty}\right)$. Then $X$ is the Shilow boundary for every Douglas algebra. For a point in $M\left(H^{\infty}\right)$, we denote by $\mu_{x}$ the representing measure on $X$ for $x$ and by supp $\mu_{x}$ the support set for $\mu_{x}$. For a function $q$ in $L^{\infty}$ (in particular if $q$ is an interpolating Blaschke product), we put $N(\bar{q})$ the closure of the union set of supp $\mu_{x}$ such that

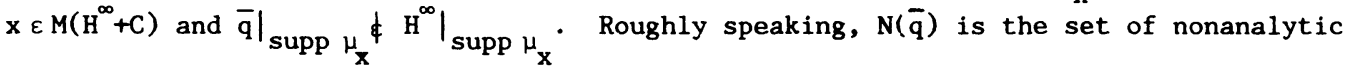
points of $q$. Set $Q C=H^{\infty}+C \cap \overline{H^{\infty}+C}$ and for $x_{0}$ in $X$, let $Q_{x_{0}}=\left\{x \in X: f(x)=f\left(x_{0}\right)\right.$ for $\tilde{\mathbf{I}} \varepsilon Q C\} . Q_{x_{0}}$ is called the $Q C$-level set for $x_{0}[9]$. For an inner function $q$, $K$. Izuchi has shown the following [5, Theorem 1(i)].

THEOREM 1. If $\mathrm{q}$ is an inner function that is not a finite Blaschke product, then,

$$
N(\bar{q})=U\left\{Q_{x} ; x \quad Z(q)\right\}
$$

In particular, the right side of 1.4 is a closed set. Now assume that $q$ is an interpolating Blaschke product, and let B be a Douglas algebra contained in $H^{\infty}[\bar{q}]$. We will always assume that $M(B) \cap Z(q)$ is not an open set in $Z(q)$, for Izuchi has shown [6] that if $B$ is a maximal subalgebra of $H^{\infty}[\bar{q}]$, then $M(B) \cap Z(q)$ is not open in $Z(q)$. We will give answers to the following two questions. When is $B$ a maximal subalgebra of $\mathrm{H}^{\infty}[\overline{\mathrm{q}}]$ or when is there a factor $\mathrm{q}_{0}$ of $\mathrm{q}$ in $\mathrm{H}^{\infty}$ such that $\mathrm{B}$ is maximal in $\mathrm{H}^{\infty}\left[\overline{\mathrm{q}}_{0}\right]$ ? These answers will be in terms of the nonanalytic points of $q$ and the invertible inner functions of $\mathrm{H}^{\infty}[\overline{\mathrm{q}}]$ that are not invertible in $\mathrm{B}$.

For a Douglas algebra $B$, we denote by $N(B)$ the closure of $U\left\{\right.$ supp $\left.\mu_{x} ; x \in M\left(H^{\infty}+C\right) / M(B)\right\}$. In particular $N\left(H^{\infty}[\bar{q}]\right)=N(\bar{q})$. In general if $A$ and $B$ are Douglas algebras such that 
$A \subseteq B$, we put $N_{A}(B)=$ the closure of $U\left\{\operatorname{supp} \mu_{x}: x \in M(A) / M(B)\right\}$ and for any inner function $b, N_{A}(\bar{b})=$ the closure of $U$ \{supp $\left.\mu_{x}: x \in M(A),|b(x)|<1\right\}$.

It is shown in [7, Corollary 2.5] that if $B \subseteq H^{\infty}[\bar{q}]$, then $N(B) \subseteq N(\bar{q})$, and it is not hard to show that $N(\bar{q}) / N(B) \geq N_{B}(\bar{q})$ (in a sense the set $N_{B}(\bar{q})$ is generated by the nonanalytic points $\left.M(B) / M\left(H^{\infty}[\bar{q}]\right) \subseteq M\left(H^{\infty}+C\right) / M\left(H^{\infty}[\bar{q}]\right)\right)$.

\section{OUR MAIN RESULT.}

We' 11 need the following lemma. It shows how small $M(B) / M\left(H^{\infty}[\bar{q}]\right)$ must be if $B$ is to be a maximal subalgebra of $H^{\infty}[\bar{q}]$. Let $\Omega=\{b: b$ is an interpolating Blaschke product with $\left.\bar{b} \varepsilon H^{\infty}[\bar{q}]\right\}$, and $\Omega(B)=\left\{b_{0} \varepsilon \Omega: \bar{b}_{0} \notin B\right\}$.

LEMMA 1. Let $q$ be an interpolating Blaschke product and $B$ be a Douglas algebra contained in $H^{\infty}[\bar{q}]$. Suppose for all $b_{0} \varepsilon \Omega(B)$, we have that $N_{B}(\bar{q}) \subseteq N_{B}\left(\bar{b}_{0}\right)$. Then $B$ is a maximal subalgebra of $\mathrm{H}^{\infty}[\overline{\mathrm{q}}]$.

PROOF. It suffices to show that if $b \in \Omega(B)$, then $B[\bar{b}]=H^{\infty}[\bar{q}]$. Hence the only Douglas algebra between $B$ and $H^{\infty}[\bar{q}]$ that contains $B$ properly is $H^{\infty}[\bar{q}]$. It is clear that $M\left(H^{\infty}[\bar{q}]\right) \subseteq M(B[\bar{b}])$. We show that $M(B[\bar{b}]) \subseteq M\left(H^{\infty}[\bar{q}]\right)$. Now $M(B[\bar{b}])=\{m \varepsilon M(B):|b(m)|=1\}$. It suffices to show that if $m \notin M\left(H^{\infty}[\bar{q}]\right)$, then $m \notin M(B[\bar{b}])$. Let $m \varepsilon M(B)$ such that

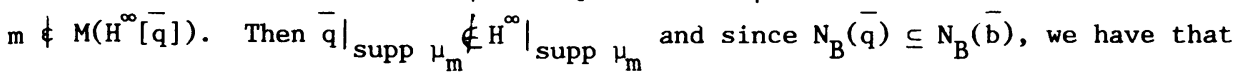
$\left.\left.\mathrm{b}\right|_{\text {supp } \mu_{\mathrm{m}}} \notin \mathrm{H}^{\infty}\right|_{\text {supp } \mu_{\mathrm{m}}}$. Thus $|\mathrm{b}(\mathrm{m})|<1$ and we get $\mathrm{m} \notin \mathrm{M}(\mathrm{B}[\overline{\mathrm{b}}])$. This shows that $M(B[\bar{b}]) \subseteq M\left(H^{\infty}[\bar{q}]\right)$, and $B$ is maximal in $H^{\infty}[\bar{q}]$.

Using Theorem 1 above, it is not hard to show directly that $N(B[\bar{b}])=N(\bar{q})$. However, by Proposition 4.1 of [7], this condition is not sufficient.

We let $E=N_{B}(\bar{q})$. This can be a very complicated set. For example, it can contain supp $\mu_{x}$ where $x$ belongs to a trivial Gleason part or a Gleason part where $|q|<1$, but yet $q \neq 0$ on this part [see 3]. So for $B$ to be maximal in $H^{\infty}[\bar{q}]$, $E$ must be as simple as possible. To see how simple, we set $\wedge(B)=\left\{b \varepsilon \Omega(B): B \subseteq H^{\infty}[\bar{b}]\right\}$ and $\wedge^{*}(B)=$

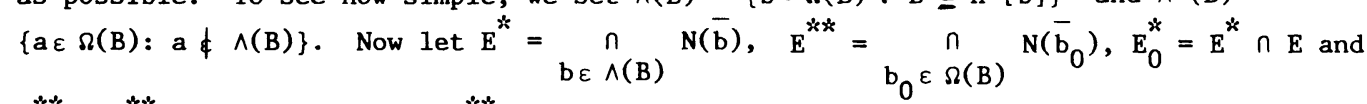
$\mathrm{E}_{0}^{* *}=\mathrm{E}^{* *} \cap \mathrm{E}$. Note that if $\mathrm{E}_{0}^{* *}=\phi$, then there are interpolating Blaschke products $\mathrm{a}_{0}$ and $a_{1}$ in $\Lambda^{*}(B)$ such that $N_{B}(\bar{q}) \cap N\left(\bar{a}_{0}\right) \cap N\left(\bar{a}_{1}\right)=\phi$. Thus we get $B \subset B\left[\bar{a}_{0}\right] \subset H^{\infty}[\bar{q}]$. To see this, just note that both $N_{B}(\bar{q}) \cap N\left(\bar{a}_{0}\right) \neq \phi$ and $N_{B}(\bar{q}) \cap N\left(\bar{a}_{1}\right) \neq \phi$ since $a_{0}$ and $a_{1}$ belong to $\Lambda^{*}(B)$. Since their intersection is empty, there is an $x_{1} \varepsilon M(B)$ such that

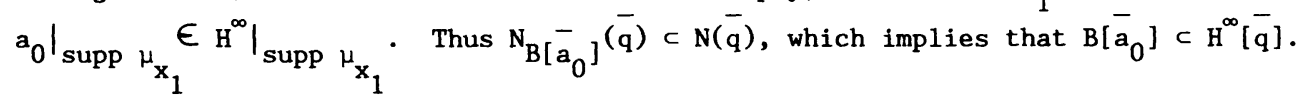
Obviously, $B \subset B\left[\bar{a}_{0}\right]$, so $B$ cannot be maximal in $H^{\infty}[\bar{q}]$ unless $E_{0}^{* *} \neq \phi$. We now state. PROPOSITION 1. Let $B$ be a Douglas algebra properly contained in $H^{\infty}[\bar{q}]$, and suppose $\mathrm{E}_{0}^{* *} \neq \phi$. Then the following statements are equivalent:

(i) $\mathrm{N}(\mathrm{B})=\mathrm{N}(\overline{\mathrm{q}})$;

(ii) $B$ is a maximal subalgebra of $\mathrm{H}^{\infty}[\overline{\mathrm{q}}]$;

(iii) $\mathrm{E}_{0}^{* *}=\mathrm{E}_{0}^{*}=\mathrm{E}$;

(iv) $\quad E_{0}^{* * *}=N_{B}(\bar{q})$. 
PROOF. We prove the following: $(i) \rightarrow($ ii $) \rightarrow($ iii $) \rightarrow($ iv $) \rightarrow($ ii $) \rightarrow($ i $)$. Suppose (i) holds. We will show that $N_{B}(\bar{q}) \subseteq N_{B}(\bar{b})$ for all $b \in \delta(B)$. Using Lemma 1 , this will prove that $B$ is a maximal subalgebra of $H^{\infty}[\bar{q}]$. Let $b \varepsilon \Omega(B)$ and consider the Douglas algebra $B[\bar{b}]$. We have $B \subseteq B[\bar{b}] \subseteq H^{\infty}[\bar{q}]$, hence $N(B)=N(B[\bar{b}])=N(\bar{q})$. Now $N(\bar{q})=$ $N(B) \cup N_{B}(q)$, so by the above equality we have that $\left.N_{B}(\bar{q}) \leq N(B \mid \bar{b}]\right)$. Thus, if $\times \varepsilon M(B)$

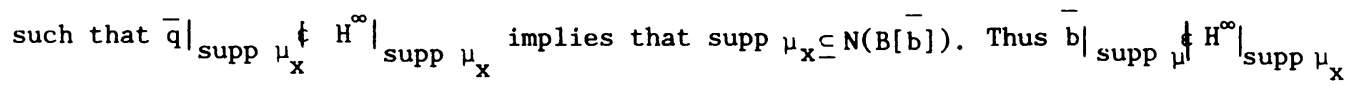
and $N_{B}(\vec{q}) \subseteq N_{B}(\bar{b})$. We have $(i) \rightarrow$ (ii).

Next suppose that $(i i)$ holds. It is clear that $E_{0}^{* *} \subseteq E_{0}^{*} \subseteq E$. We must show that $E_{0}^{*} \mid E_{0}^{* *}$ and $E \mid E_{0}^{*}$ are empty sets. First we show that $E_{0}^{*} \mid E_{0}^{* *}$ is empty. Suppose not. Then there is an $x \in M(B)$ and $a b_{0} \varepsilon \Lambda^{*}(B)$ such that $\left.\left.\bar{b}_{0}\right|_{\text {supp }} \mu_{x} H^{\infty}\right|_{\text {supp }} \mu_{x}$ and supp $\mu_{\mathrm{x}} \subseteq \mathrm{E}_{0}^{*}$. It is clear by Theorem 1 that supp $\mu_{\mathrm{x}} \cap \mathrm{N}\left(\overline{\mathrm{b}}_{0}\right)=\mu_{\phi}$. Consider the algebra $B\left[\bar{b}_{0}\right]$. Since $b_{0} \varepsilon \Lambda^{*}(B), E \subseteq B\left[\bar{b}_{0}\right]$. Since supp $\mu_{x} \subseteq N(\bar{q})$ and supp $\mu_{x} \pm N\left(\bar{b}_{0}\right)$, we have that $\left|b_{0}(x)\right|=1$, so we have supp $\mu_{x} \subseteq N(\bar{q}) / N_{B}\left[\bar{b}_{0}\right](\bar{q})$. This implies that $B\left[\bar{b}_{0}\right] \subseteq H^{\infty}[\bar{q}]$, which is a contradiction. So $\mathrm{E}_{0}^{* *}=\mathrm{E}_{0}^{*}$.

Now we show that $E / E_{0}^{*}$ is empty. Again suppose not. Hence there is a $y \in M(B)$ such that supp $\mu_{y} \subseteq E$, but supp $\mu_{y} \subseteq E_{0}^{*}$. There is a b $\in \Lambda(b)$ such that supp $\mu_{y} \subseteq N(\bar{b})$.

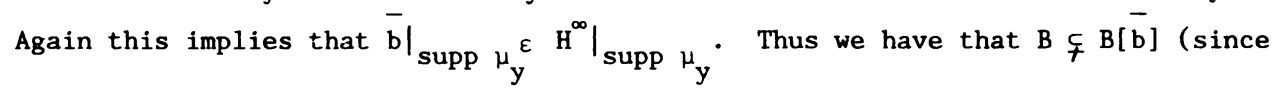
$b \in \Lambda(B)$ ) and $B[\bar{b}] \subsetneq H^{\infty}[\bar{q}]$ (since supp $\mu_{y} \subseteq N(\bar{q}) / N_{B}[\bar{b}](\bar{q})$ ), which is a contradiction. So we get $\mathrm{E}_{0}^{*}=\mathrm{E}$. This shows that $(\mathrm{ii}) \rightarrow$ (iii).

It is trivial that if (iii) holds, $E_{0}^{* *}=N_{B}(\bar{q})$.

If (iv) holds and $\mathrm{b}$ is any interpolating Blaschke product in $\Omega(\mathrm{B})$, then by (iv) $\mathrm{N}_{B}(\bar{q}) \subseteq N_{B}(\bar{b})$ so by Lemma $1, B$ is a maximal subalgebra of $H^{\infty}[\bar{q}]$.

Finally, suppose (ii) holds. We are going to show that $N(B)=N(\bar{q})$. Suppose not. Then $N(B) \subseteq N(\bar{q})$. By Theorem 1 there is a $Q-C$ level set $Q$ with $N(B) \cap Q=\phi$. Put $B_{0}=\left[H^{\infty}, \bar{I} ; I\right.$ is an interpolating Blaschke product with $\bar{I} \varepsilon H^{\infty}[\bar{q}]$ and $\left.\left.\left.\bar{I}\right|_{Q} \varepsilon H^{\infty}\right|_{Q}\right]$. By Proposition 4.1 of $[7]$, we have $B_{0} \subsetneq H^{\infty}[\bar{q}]$ and $N\left(B_{0}\right)=N(\bar{q})$. Since $N(B) \cap Q=\phi$, we also have $B \subseteq B_{0}$ (because $N(B) \subseteq N\left(B_{0}\right)$ ). This implies that $B$ is not a maximal subalgebra of $H^{\infty}[\bar{q}]$, which is a contradiction. Thus $N(B)=N(\bar{q})$.

Now suppose we have that $\mathrm{E}_{0}^{* *} \subseteq \mathrm{E}_{0}^{*} \subseteq \mathrm{E} \quad\left(\mathrm{E}_{0}^{* \dot{*}}=\phi\right.$ is possible $)$.

When is there a factor $q_{0}$ of $q$ in $H^{\infty}$ such that $B$ is a maximal subalgebra of $H^{\infty}\left[\bar{q}_{0}\right]$ ( $B=H^{\infty}\left[\bar{q}_{0}\right]$ is not possible)? To answer this question, let $\Omega_{0}=\left\{q_{0}: q^{q} q_{0} \varepsilon H^{\infty}\right\}$, and $\Omega_{0}(B)=\left\{q_{0} \varepsilon \Omega_{0}: B \subseteq H^{\infty}\left[\bar{q}_{0}\right]\right\}$.

Set $F=\underset{q_{0} \varepsilon i z_{0}(B)}{n} N\left(\bar{q}_{0}\right)$. Suppose $F=N\left(\bar{q}_{0}\right)$ for some factor $q_{0}$ of $q_{\text {in }} H^{\infty}$. Then $B \subseteq H^{\infty}\left[\bar{q}_{0}\right]$. So $q_{0}$ is our possible candidate. Next, let $\Omega_{q_{0}}=\{c: c$ is an interpolating Blaschke product with $\left.\bar{c} \varepsilon H^{\infty}\left[\bar{q}_{0}\right]\right\}$, 


$$
\begin{aligned}
& \Omega_{\mathrm{q}_{0}}(\mathrm{~B})=\Omega_{\mathrm{q}_{0}} \cap \Omega(\mathrm{B}), \Lambda_{\mathrm{q}_{0}}(\mathrm{~B})=\Omega_{\mathrm{q}_{0}}(\mathrm{~B}) \cap \Lambda(\mathrm{B}), \Lambda_{\mathrm{q}_{0}}^{*}(\mathrm{~B})=\Omega_{\mathrm{q}_{0}} \text { (B) } \cap \Lambda^{*}(\mathrm{~B}), \\
& \mathrm{F}_{0}=\mathrm{E} \cap \mathrm{N}\left(\overline{\mathrm{q}}_{0}\right), \mathrm{F}^{*}=\mathrm{E}_{0}^{*} \cap \mathrm{F}, \mathrm{F}^{* *}=\underset{\mathrm{c} \varepsilon \Omega_{\mathrm{q}_{0}}(\mathrm{~B})}{\mathrm{N}(\mathrm{c}), \mathrm{F}_{0}^{*}=\mathrm{F}^{*} \cap \mathrm{F}_{0} \text {, and finally }} \\
& \mathrm{F}_{0}^{* *}=\mathrm{F}^{* * *} \cap \mathrm{F}_{0} .
\end{aligned}
$$

We have the following.

COROLLARY 1. Let $\mathrm{q}_{0}$ be a factor of $\mathrm{q}$ in $\mathrm{H}^{\infty} \underline{\text { such }} \underline{\text { that }} \mathrm{F}=\mathrm{N}\left(\overline{\mathrm{q}}_{0}\right)$ and assume $\mathrm{F}_{0}^{* *} \neq \phi$. If any of the following conditions hold:

$$
\begin{aligned}
& \text { (i) } \mathrm{F}_{0}=\mathrm{F}_{0}^{*}=\mathrm{F}_{0}^{* * *} \\
& \text { (ii) } \mathrm{F}_{0}^{* *}=\mathrm{N}_{\mathrm{B}}\left(\mathrm{H}_{0}\right) \text {, where } \mathrm{H}_{0}=\operatorname{n}_{\mathrm{q}_{0} \varepsilon \Omega(\mathrm{B})} \mathrm{H}^{\infty}\left[\mathrm{q}_{0}\right] \text {. }
\end{aligned}
$$

Then $B$ is a maxima subalgebra of $H_{0}=H^{\infty}\left[\bar{q}_{0}\right]$ where $q_{C} \varepsilon \Omega_{0}(B)$.

The fact that $F=N\left(q_{0}\right)$ for some $q_{0} \varepsilon \Omega_{0}(B)$ implies that $H_{0}=H^{\infty}\left[\bar{q}_{0}\right]$ and our corollary follows from Proposition 1.

We now consider this question for the genral Douglas algebras. Let $A$ and $B$ be Douglas algebras such that $A \subseteq B$ and there is an inner function $q$ with $B \subseteq A[\bar{q}]$. When this occurs we say that $A$ is near $B$. It is well known that if $B=L^{\infty}$ and $A$ is any Douglas algebra properly contained in B, then $A$ is not near $B$, that is, $B \subseteq A[\bar{q}]$ for any inner function $q$. In fact $L^{\infty}$ is not countably generated over any Douglas algebra A [10]. So by the results of C. Sundberg [10] any Douglas algebra B which is countably generated over $A$ is also near it.

The following result comes from [2, Lemma 5] and gives equivalent conditions for two Douglas algebras to be near each other [see 11, Theorem 1 for a similar result].

THEOREM 2. Let $A$ and $B$ be Douglas algebras with $H^{\infty}+C \subsetneq A \subseteq B$ and 9 be an inner function. Then the following statements are equivalent.

$$
\begin{aligned}
& \text { (i) } M(A)=Z_{\dot{A}}(q) \cup M(B) \\
& \text { (ii) } \phi B \subseteq A
\end{aligned}
$$

where $Z_{A}(q)=Z(q) \cap M(A)$.

PROOF. Assume ( $i$ ) holds; we show that $\phi B \subseteq A$. Let $b$ be any interpolating Blaschke product for which $\bar{b}$ is in $B$. If $x$ is in $Z_{A}(b)$, we show that $x$ is also in $Z_{A}(q)$. Now $x$ is in $M(A)$ and $b(x)=0$ implies that $x$ is not in $M(B)$, since $\bar{b}$ is in $B$. So by (i) we have that $x$ must be in $Z_{A}(q)$. Thus $Z_{A}(b) \subseteq Z_{A}(q)$, and by Theorem 1 of [4] we have $\bar{b}$ is in $A$. Now let $f$ be any function in $B$. By the Chang Marshall Theorem $[1,8]$ there is a sequence of functions $\left\{h_{n}\right\}$ in $H^{\infty}$ and a sequence of interpolating Blaschke products $\left\{b_{n}\right\}$ with $\bar{b}_{n} \in B$ for all $n$, such that $h_{n} \bar{b}_{n} \rightarrow f$. But $h_{n}\left(\bar{b}_{n}\right) \rightarrow f$ belongs to $A$ since $b_{n}$ is in A for all $n$. This proves (ii). 
Assume ( $i i)$ holds. Let $x$ be in $M(A)$ but not in $M(B)$. Then there is an inner function $b$ which is invertible in $B$ such that $|b(x)|<1$. For any positive integer $n$, the function $f_{n}=q \vec{b}^{n}$ is in $A$, so

$$
|g(x)|=|b(x)|^{n}\left|f_{n}(x)\right| \leq|b(x)|^{n} .
$$

Letting $\mathrm{n} \rightarrow \infty$ we get $(\mathrm{x})=0$. This proves $(\mathrm{i})$.

Set $Z_{B}(q)=M(B) \cap Z_{A}(q)$ and $Z_{B}^{*}(q)=Z_{A}(q) / Z_{B}(q)$; then $M(A) / M(B)=\underset{x \varepsilon Z_{B}^{*}(q)}{U} P_{x}$, since $M(A)=M(B) \cup Z_{A}(q)$.

As we have previously done, let $\Omega(B, A)$ be the set of interpolating Blaschke products $b$ such that $b \varepsilon B$ but $b \notin A$ and set $W^{*}=\prod_{b \in \Omega(B, A)} N_{A}(\bar{b})$. We assume $W^{*} \neq \phi$.

Using Proposition 1, Theorem 2 and Lemma 1, we have the following result.

PROPOSITION 2. Let A and B be arbitrary Douglas algebras such that $A$ is near $B$. Then the following statements are equivalent:

$$
\begin{aligned}
& \text { (i) } N_{A}(B) \subseteq N_{A}(\bar{b}) \text { for all } b \in \Omega(B, A) \text {; } \\
& \text { (ii) A is a maximal subalgebra of } B \text {; } \\
& \text { (iii) } w^{*}=N_{A}(B) \text {. }
\end{aligned}
$$

PROOF. Assume that (i) holds. Since $A$ is near to $B$, there is an inner function

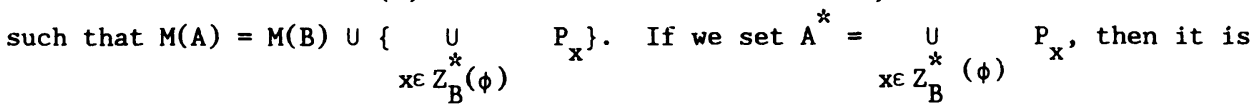
immediate that

$$
N_{A}(B)=\text { closure of } U\left\{\operatorname{supp} \mu_{x}: x \in A^{*}\right\}
$$

Let $b$ be any alement in $\Omega(B, A)$. By (i) we have that $N_{A}(B) \subseteq N_{A}(\vec{b})$. As in proof of Lemma 1 we have that $A[\bar{b}]=B$. Thus is a maximal in $B$.

Assume that (ii) holds, and let $\times \varepsilon A^{*}$. Since $A$ is near $B$, we have that $M(A)=$ $M(B) \cup A^{*}$. If $b \in \Omega(B, A)$, then by our hypothesis $A[\bar{b}]=B$, which implies that if $y \in M(A)$ and $|b(y)|=1$, then $y \in M(B)$ (since $M(A[\bar{b}])=\{g \in M(A):|b(g)|=1\}=M(B)$ ).

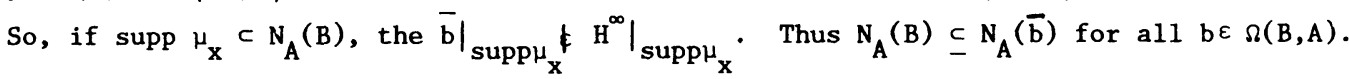
This implies that $\mathrm{N}_{\mathrm{A}}(\mathrm{B}) \subseteq \mathrm{W}^{*}$

To show what $W^{*} \subseteq N_{A}(B)$, let $b \in \Omega(B, A)$. Hence $\bar{b} \varepsilon B$; therefore we have

$$
\begin{aligned}
N_{A}(\bar{b}) & =\text { closure of } U\left\{\operatorname{supp} \mu_{x} ; x \in M(A),|b(x)|<1\right\} \\
& =\text { closure of } U\left\{\operatorname{supp} \mu_{x} ; x \in M(A) / M(B), \mid b(x)<1\right\} \\
& \subseteq \text { closure of } U\left\{\operatorname{supp} \mu_{x} ; x \in M(A) / M(B)\right\} \\
& =N_{A}(B) .
\end{aligned}
$$


Since this is true for any $b \varepsilon \Omega(B, A)$, we have $N_{A}(B) \geq W^{*}$. Thus $W^{*}=N_{A}(B)$ if $A$ is maximal in $B$.

It is trivial that if (iii) holds, $N_{A}(B) \subseteq N_{A}(\bar{b})$ for all $b \in \Omega(B, A)$. We are done.

In Proposition 4.1 of [7] Izuchi constructed a family of Douglas algebras B contained in $\mathrm{H}^{\infty}[\overline{\mathrm{q}}]$ with the property that $\mathrm{N}(\mathrm{B})=\mathrm{N}(\overline{\mathrm{q}})$. By Proposition 1 , we have that this family is a family of maximal subalgebras of $\mathrm{H}^{\infty}[\overline{\mathrm{q}}]$.

Finally we close this paper with the following question that $I$ have been uable to answer.

QUESTION 1. Recall that if $q$ is an interpolating $B$ laschke product, then $N(\bar{q})=N(B) \cup N_{B}(\bar{q})$ for any Douglas algebra with $B \subseteq H^{\infty}[q]$. Does there exist a Douglas algebra $B_{0} \subseteq H^{\infty}[\bar{q}]$ with $N_{B_{0}}(\bar{q})=N(\bar{q})$ ?

\section{REFERENCES}

1. CHANG, S.Y. A Characterization of Douglas Subalgebras, Acta. Math. 137 (1976) 81-89.

2. GUILLORY, C.J. Lemmas on Thin Blaschke Products and Nearness of Douglas Algebras, Preprint.

3. GUILLORY, C.J. Douglas Algebras of the form $\mathrm{H}^{\infty}[\overline{\mathrm{q}}]$, to appear in J. Math Anal. \& App1., 1987.

4. GUILLORY, C.J., IZUCHI, K., and SARASON, D. Interpolating Blaschke Products and Division in Douglas Algebras, Proc. Royal Irish Acad. Sect., A84 (1984) 1-7.

5. IZUCHI, K. QC-Level Sets and Quotients of Douglas Algebras, J. Funct. Anal., 65 $(1986), 293-308$.

6. IZUCHI, K. Zero sets of Interpolating Blaschke Products, Pacific J. Math., $19(1985), 337-342$.

7. IZUCHI, K. Countably Generated Douglas Algebras, To appear in Trans. Amer. Math. Soc.

8. MARSHALL, D. Subalgebras of $\mathrm{L}^{\infty}$ containing $\mathrm{H}^{\infty}$, Acta Math. 137 (1976), 91-98.

9. SARASON, D. The Shilov and Bishop decomposition of $\mathrm{H}^{\infty}+\mathrm{C}$, Conference on Harmonic Analysis in Honor of A. Zygmund, in Wadsworth Math Series, pp. 461-474, California, 1981 .

10. SUNDBERG, $\mathrm{C}$. A Note on Algebras between $\mathrm{L}^{\infty}$ and $\mathrm{H}^{\infty}$ Rocky Mountain $\mathrm{J}$. Math. $\underline{11}$ 2 (1981), 333-335.

11. YOUNIS, R. Division in Douglas Algebras and Some Applications, Arch. Math., Vol. $45,555,560$. 


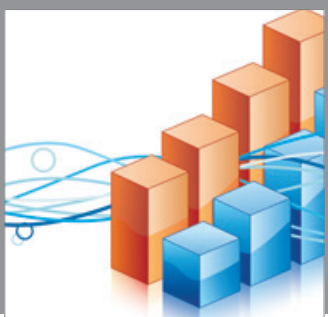

Advances in

Operations Research

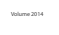

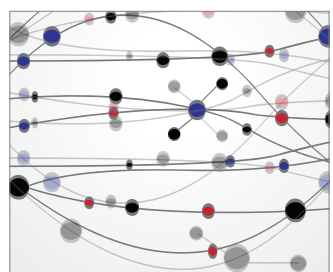

\section{The Scientific} World Journal
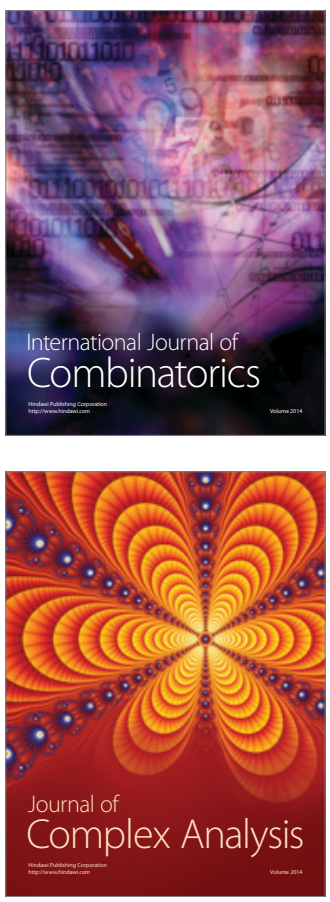

International Journal of

Mathematics and

Mathematical

Sciences
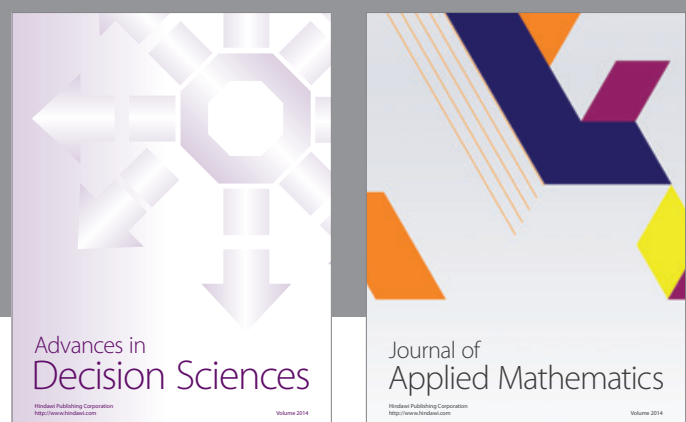

Journal of

Applied Mathematics
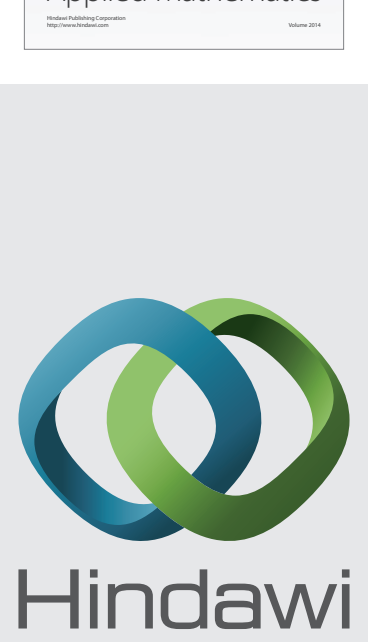

Submit your manuscripts at http://www.hindawi.com
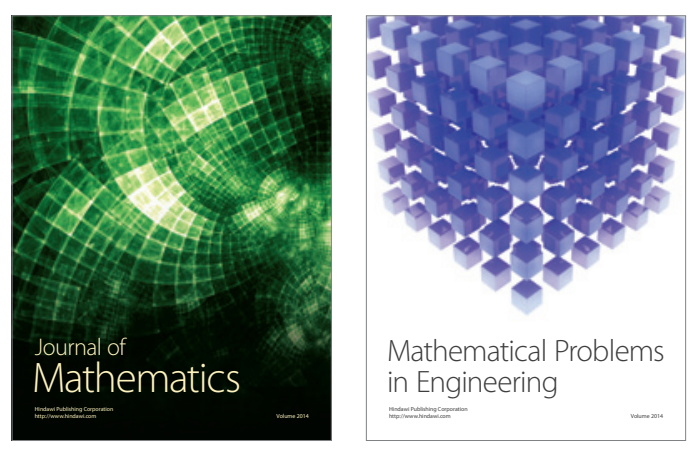

Mathematical Problems in Engineering
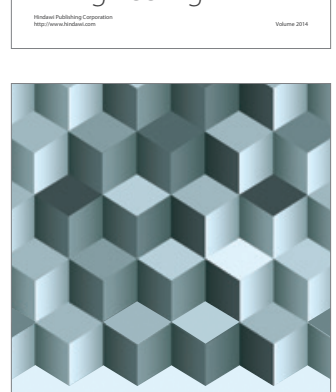

Journal of

Function Spaces
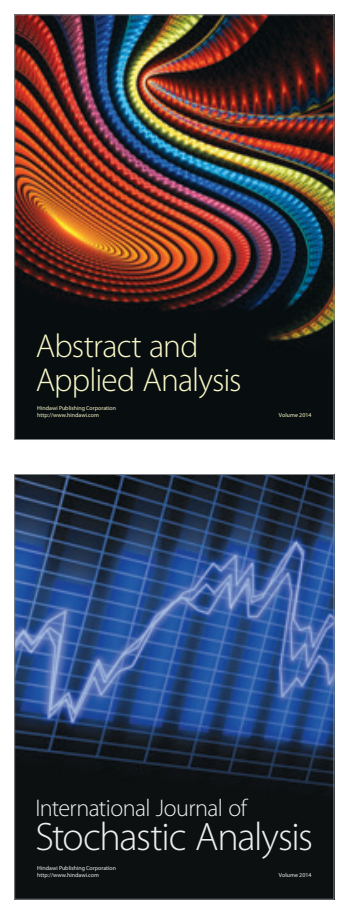

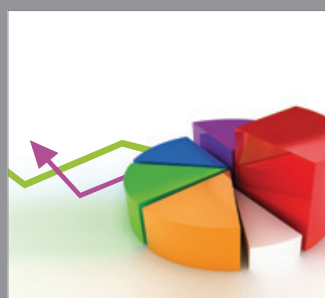

ournal of

Probability and Statistics

Promensencen
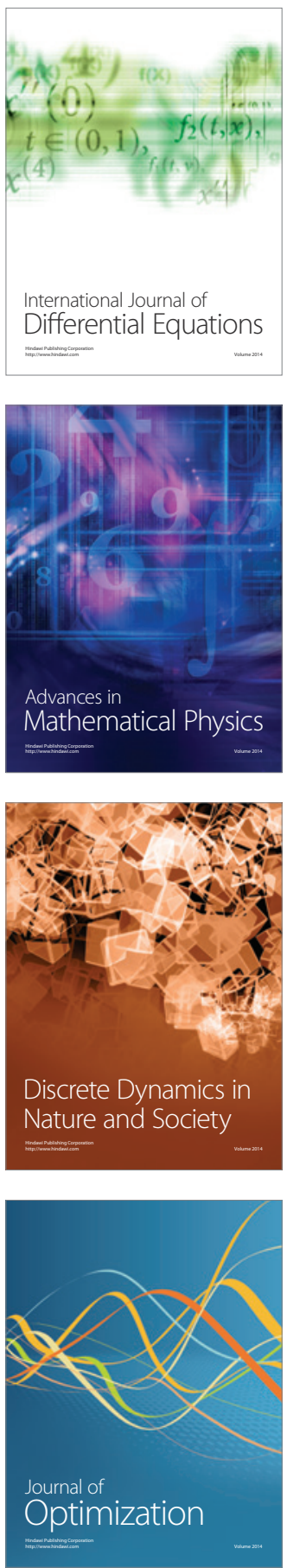